\title{
Establishing the Brand-New Trans-Graduate Education Frame: Challenges of Hokkaido University "Nitobe School Program"
}

\author{
Yasuhiro Yamanaka *, Michiyo Shimamura ${ }^{\dagger}$,
}

\begin{abstract}
To correspond to a drastic change in international society such as "globalization," graduate education plays a key role in development of human resources to nurture competencies such as transferable skills as well as their advanced specialties. We report a new trans-graduate-school educational program called "Nitobe School" in Hokkaido University, which has unique mechanisms: "3+1 Competencies," "Microcosm of international society," "NITOBE Portfolio," and "Systems of Mentors and Advisors." Nitobe School gives students supportive push to respect and understand different discipline, nationalities, cultures, and discussing in suitable language. Especially, "Microcosm of international society," i.e. students who have various backgrounds learn together in a classroom, works well for the students for whom English is not the first language and discuss with diverse students. We spent one year to prepare the coursework as "microcosm of international society" and applied many different mechanisms for promoting students' proactive learning and quality assurance of Nitobe School program. To make a success of "microcosm of international society," it is required for professors as well as students that deeply respect of other people and understanding of diversity. With these skills and understandings, a good platform can be built for faculty development and student guidance.
\end{abstract}

Keywords: Competencies, Graduate School, Graduate Education Reform, Team-Based Learning,

\section{Introduction}

Research and course works are inseparable in graduate schools. It has been traditionally considered that the advanced expertise required by professions are unintentionally equipped while pursuing his or her master or doctorial degrees, because of the statement as "Graduate

\footnotetext{
* Faculty of Environmental Science, Hokkaido University, Hokkaido, Japan

$\dagger$ Innovation Promotion Office via Marine-Earth Science and Technology,Japan Agency for Marine-Earth

Science and Technology, Kanagawa, Japan
} 
Schools are designed to teach and study academic theories and applications, and inquire deeply into them or cultivate knowledge and outstanding ability in depth to engage in professions which require advanced expertise, while contributing to the development of culture." is laid down in Paragraph 1 of Article 99 of the School Education Law in Japan [1].

The present society demands graduate schools to develop human resources that correspond to a drastic change in international society such as "globalization." After World War II, Japan has developed industries providing high-quality products to the world by promoting improvements such as Toyota's Just-In-Time manufacturing, "Kanban system" [2]. To correspond to the industry needs, Japanese universities have also played a role of supplying human resources to the industries. However, such iterative improvements cannot perform for the rapid changes under the "globalization" with various senses of values. It means "A good product sells without explanation" is not accepted anymore in global society. Industries even in Japan are required to change into innovative companies such as Apple and Google. As a team having highly various specialties, specialists should innovatively work by communicating to others with different specialties. Under the various scenes and circumstances, specialists should explain and use their specialties. Even in academia, specialists will often be requested to explain their specialties in international academic meetings and collaborative research. Therefore, in the present scenario, specialists are supposed to acquire not only their respective specialties but also acquire competencies such as abilities that stimulate their specialties.

To correspond to the present social demand, the Ministry of Economy, Trade and Industry (METI), Japan has defined competencies required for the working people as "Fundamental Competencies for Working Persons" in 2006 [3], and the Ministry of Education, Culture, Sports, Science and Technology (MEXT), Japan also defined competencies required for undergraduates as Gakushiryoku (Bachelor's competencies) in 2008 [4]. While the METI's competencies define the good businessperson in the labor market, MEXT's competencies define the highly educated person should have such as social responsibility as good citizens, and attitude of the lifelong learning.

Graduate students are also required to have these competencies along with their advanced expertise, and it should be higher level than undergraduates' one. To make higher education corresponding with "globalization", MEXT has also secured budgets to reform universities through projects such as the "Top Global University Project" [5]. Hokkaido University in Japan was adopted as a university of Type A in the "Top Global University Project."

The "d.school" established in Stanford University in 2005 [6] is well known as an education program helping students to develop their creativities, which corresponds to the innovative companies such as Apple and Google. In Japan, "i.school" in the University of Tokyo was established in 2009 for students to be innovative people [7]. In these schools, students and faculties coming from different graduate schools make mutually teaching and learning such as a method of Project-Based Learning (PBL) as an interdisciplinary program. From 2008, National Institute of Advanced Industrial Science and Technology (AIST) also hosted "Innovation School," which offered curriculum for postdoctoral researchers and Ph.D. students to encourage broad their perspectives and raise their awareness [8].

Haworth and Conrad proposed engagement theory of quality graduate education to evaluate and improve higher education programs [9]. Their definition of "engagement" is meant to describe a "high quality programs... in which students, faculty, and administrators invest significant time and effort in mutually supportive teaching and learning", and their theory is involving: (1) diverse and engaged participants, (2) participatory cultures, (3) interactive teaching and learning, and (4) adequate resources [10]. This theory was used to evaluate graduate programs, for ex- 
ample, interdisciplinary graduate programs [10] and accredited counselor education program [11].

Hokkaido University was established in 1876 as Sapporo Agriculture College, and currently has around 6,000 students in 18 graduate schools (including professional graduate schools) in 2015, and has adopted and cultivated as its ethos the four basic philosophies of "frontier spirit," "global perspectives," "all-round education," and "practical learning." In 2014, Hokkaido University planned out "Future Strategy for the $150^{\text {th }}$ Anniversary of Hokkaido University" [12], in which the educational strategy is described to be such that "Hokkaido University will produce graduates who will play a leading role in contributing to the development of the global society. As specialists in their fields, they will possess sound judgment and deep insight, along with the ability to understand and communicate with different cultures."

In the academic year 2013, a group of interested professors from various graduate schools started designing a new education program with trans-graduate school style to foster students who have abilities activating their specialties. In the academic year 2014, the official committee of preparing Nitobe School (its chair is the vice-president in charge of education) took over its aim from the original group, and made their idea into "Fundamental Concept of Nitobe School." They regarded the establishment of Nitobe School as the first step to the educational reformation toward the redefining competencies that students obtain in their graduate schools. In fact, they had the plan to assess courses provided by each graduate schools and certify each course as the associate courses of Nitobe School in which students obtain the specific part of " $3+1$ competencies" (as described latter). This idea did not include into the final version of "Fundamental Concept of Nitobe School" due to the objection by executive vice president taking charge of education. However, Nitobe School has the role of a prototype of graduate education promoting the educational reform of each graduate school.

Nitobe was named after Dr. Inazo Nitobe (1862-1933) who graduated Sapporo Agriculture College and had an international success as Under-Secretaries General of the League of Nations. Nitobe School program became one of main educational component of "Top Global University Project" in Hokkaido University. Nitobe School's capacity per year was set 60 graduate students in the basic program for the first academic year of 2015 (and are to be 120 students basic program and 25 students in the advanced program after the academic year 2017). The president of Hokkaido University takes charge as the principal of Nitobe School.

In this paper, we provide the concept of new trans-graduate-school education program "Nitobe School" in Hokkaido University as a case study of education program in which graduate students acquire competencies. In section 2, the competencies cultivated in Nitobe School namely, "3+1 Competencies," learning environment produced by Nitobe School, "Microcosm of international society," and mechanisms promoting students' proactive learning and quality assurance of Nitobe School are introduced. We also discuss them based on engagement theory of quality graduate education. In section 3, we also present the current status and issues again, two years after the start of Nitobe School.

\section{Fundamental Concept of Nitobe School}

\subsection{Abilities Demanded by Society: “3+1 Competencies"}

The present society emphasis on graduate schools to foster education such that graduates can acquire the following abilities along with their own specialties obtained in their respective 
graduate schools: (1) the ability to continuously self-improve through acquirement of higher-level specialty against multifaceted problems, (2) the ability of playing a leading role in a specialists team to resolve these problems, (3) the ability to create socially new values and innovative approach to strengthen and improve society. Along with the above mentioned abilities, it is also important to obtain social credibility supported by $(+1)$ professional ethics. Nitobe School defines these abilities as " $3+1$ competencies" and this is the core of this program, as follows.

\section{(1) Continuous Personal Development:}

Graduates create visions for their future and identify the problems they seek to resolve. Graduates tackle challenges to achieve set objectives and continuously improving themselves by modifying the plans as necessary based on an appropriate understanding of their abilities and the circumstances. Its competency elements are:

(1.1) Curiosity and desire for self-improvement: These comprise the desires to explore the unknown, improve the present, and take actions toward self-improvement based on one's future visions.

(1.2) Challenging new horizon: This is the willingness to take on new challenges, to try again and keep trying until one succeeds.

(1.3) Self-knowledge: This is the understanding of one's own strengths and weaknesses in light of one's objectives or challenges.

(1.4) Predictive capability: This is the ability to predict future events and foresee upcoming needs based on one's comprehensive understanding of past developments and the present situation.

(1.5) Abilities of planning and execution: These mean providing an optimal roadmap for achieving a goal and steadily taking the steps according to the roadmap.

(1.6) Self-management: This is to identify necessary improvements based on the results of implementing a plan and to modify the plan by incorporating the improvements for enhanced efficiency. It may also involve redefining issues to achieve goals.

\section{(2) Team Organization and Management:}

Graduates work independently as professionals to achieve the ultimate goals that you share with the other team members. They and other team members trust and collaborate with one another to achieve the best possible results by effectively obtaining and allocating resources, reconciling differences and avoiding interpersonal conflicts. And they maintain the optimal conditions for their team. Its competency elements are:

(2.1) Ability to articulate and share one's vision: This is the ability to clearly understand the ultimate goals to such an extent that one can share them with other team members and articulate them from various perspectives to ensure appropriate understanding by other team members. It is also the ability to take responsibility for what one says and to share the common goals and anticipated results with other team members.

(2.2) Mutual understanding of the professionality: This means a proper understanding of the abilities and characteristics of others' professionality. It also means the clear explanation of their professionality to others.

(2.3) Networking skills: These are the skills to identify connections to build a required team of professionals.

(2.4) Trust-building ability: This is the ability to build relationships of trust with collaborators. With this skill, it is highly possible to reproduce the expected behavior.

(2.5) Collaborative skills: These are the skills to work independently as a professional to achieve shared goals with other team members and to produce the maximum team 
results while drawing out one another's strengths and compensating for one another's shortcomings.

(2.6) Abilities to obtain/organize resources: These are the abilities to procure necessary resources or reallocate/sort out resources to use them efficiently.

(2.7) Preparatory negotiation skills: These are the skills to identify differences of opinions and/or interpersonal conflicts among the concerned parties beforehand, and to share information and coordinate opinions.

(2.8) Mediation skills: These are the skills to identify the causes of any interpersonal conflicts and to create a new environment to achieve goals.

\section{(3) Giving Back to Society:}

Benefit society in one's professional role by proposing a better direction and by working in that direction based on one's recognition of the needs at hand to deliver the maximum wealth and wellbeing to society. Its competency elements are:

(3.1) Abilities to contribute to society and social wellbeing: These abilities mean considering and trying to contribute to the wealth and wellbeing of society at large.

(3.2) Recognition of needs: This means considering current challenges and issues and grasping the needs of others and society at large.

(3.3) Awareness of social roles: This means recognizing one's standing and expected roles in society.

(3.4) Ability to propose solutions: This is the ability to work out solutions to social problems and to propose them in a readily acceptable form.

(3.5) Ability of making decisions: This is the ability to select the best of all available options in one's professional capacity in consideration of the levels of significance and urgency. It also involves taking responsibility for making the decision.

(3.6) Resilience: This is the ability not to give up easily to achieve one's goals, and involves flexibility.

\section{(+1) Professional Ethics:}

Make fair and impartial decisions and take responsibility for your decisions in your role as a highly-educated professional in consideration of the interests of society at large and the impacts on it instead of only paying attention to your own interests. Its competency elements are:

(+1.1) Consideration: This means looking at things from various perspectives, not only one's own viewpoint. It also means having the imagination to look at things from another person's perspective.

(+1.2) Risk management: This means predicting risks and acting based on appropriate judgment.

(+1.3) Integrity: This means never telling a lie to oneself or others.

(+1.4) Ethical sensitivity: This means being sensitive to ethical issues. It means, for example, to be conscious of human rights, environmental issues, and issues concerning harassment, racial discrimination, gender, privacy and waste, as well as having the imagination to consider other people's emotions.

(+1.5) Ethical reasoning: This is the ability to articulate the grounds for ethical judgments, rationally explain the reasons for reaching conclusions and share related processes.

(+1.6) Balance of fairness, impartiality and efficiency: These guarantee equal opportunities and honesty. They also involve efforts to achieve optimum results with minimal resources.

(+1.7) Distinguish of normative and culture: This means distinguishing ethical issues from cultural ones. 
The "d.school" presents eight abilities: (1) navigate ambiguity, (2) learn from others, (3) synthesize information, (4) experiment rapidly, (5) move between concrete and abstract, (6) build and craft intentionally, (7) communicate deliberately, and (8) design your design work, and the "i.shool" presents five guiding principles: (1) human-centered Innovation, (2) structuring knowledge for creative thinking, (3) social issues into innovation opportunities, (4) cultivating new leadership, (5) providing real experiences. These abilities or principles in two schools seem relevant to components how to make innovation by a team of specialists in a short-termed period with high efficiency, which mainly correspond to our "Team Organization and Management" and some correspond to a part of "Giving Back to Society". On the other hand, we regarded "Continuous Personal Development" can be the most important competencies among " $3+1$ competencies" in one's life. We also regard "Professional Ethics" as a responsibility of highly educated leaders should have, and it is the antithesis of globalization that reduces the diversity of international society. By the analogy of the relationship between METI's "Fundamental Competencies for Working Persons" and MEXT's Bachelor's competencies, we may regard the relationship between the abilities/principles in two schools and " $3+1$ competencies" as the relationship between practical skills for innovation and "Doctor's/Master's competencies" activating student's professionality (as described later).

We developed and confirmed our concept of " $3+1$ competencies" through discussions with various specialists from outside of the university in the aspects of academic and industrial communities, internationalization. For example, we held an international symposium titled "Reform of Graduate Education: How to Develop Students' Competencies and Their Specialties" on $19^{\text {th }}$ in February 2015. In the symposium, Prof. Motohisa Kaneko suggested an ideal form of the Graduate Educations, Mr. Kuniaki Sato explained the roles of graduate schools accredited by MEXT, Ms. Tomoko Hasegawa showed expectations of graduate education from the business community investigated by Japan Business Federation, and three Professors, Katherine S. Newman, Dahrul Syah, Seksom Attamangkune reported the current trends in graduate education in the United States, Indonesia and Thailand, respectively.

\section{2 "Microcosm of international society" and team-based learning}

Nitobe School created a learning environment named "microcosm of international society", that is the style of students learning in a same class room with other students with different background like discipline, nationalities, cultures, and languages, using the advantage of Research University. Nitobe School adopts team-based learning (TBL), in which multiple teams are composed of students with different backgrounds and skills (Fig. 1). TBLs were adopted in courses that many students from various disciplines participate in learning consensus building for environmental conservation (e.g., a comprehensive course Introducing Environmental Science in Graduate School of Environmental Science in Hokkaido University [13]). TBL will make the student's specialties improve as well as strengthen the " $3+1$ competencies". That means each student is a beginner in one's graduate school, but also needs to be a specialist in "microcosm of international society", because the student possibly will be only one specialist in his field in that team (as a result, students must study their specialties up to expected level in that team). It is our answer of graduate level TBL in our framework, although TBL is generally regarded as a kind of teaching method that students can effectively learn contents in a well-defined coursework. From our graduate education experience, the students spontaneously acquired the " $3+1$ competencies" and were able to understand various social issues from global 
to local scales through solving the problems for which an answer cannot easily be found, such as global issues listed by Sustainable Development Goals (SDGs) of United Nations in 2015 [14].

Our "microcosm of international society" is perfectly matched Haworth and Conrad's engaged theory of quality graduate education: diverse and engaged participants, and participatory cultures. Especially, "microcosm of international society" realized the high diversity of nationalities, cultures, and languages as well as disciplines. Different perspectives by students participating in "microcosm of international society" are also an important part of the interdisciplinary cooperation, though they complicate communication [15]. Our adapted learning style of TBL and various contents such as SDGs are suitable for quality graduate education suggested by Haworth and Conrad. Especially, TBL was an excellent learning style adapted by "d.school" and "i.school."

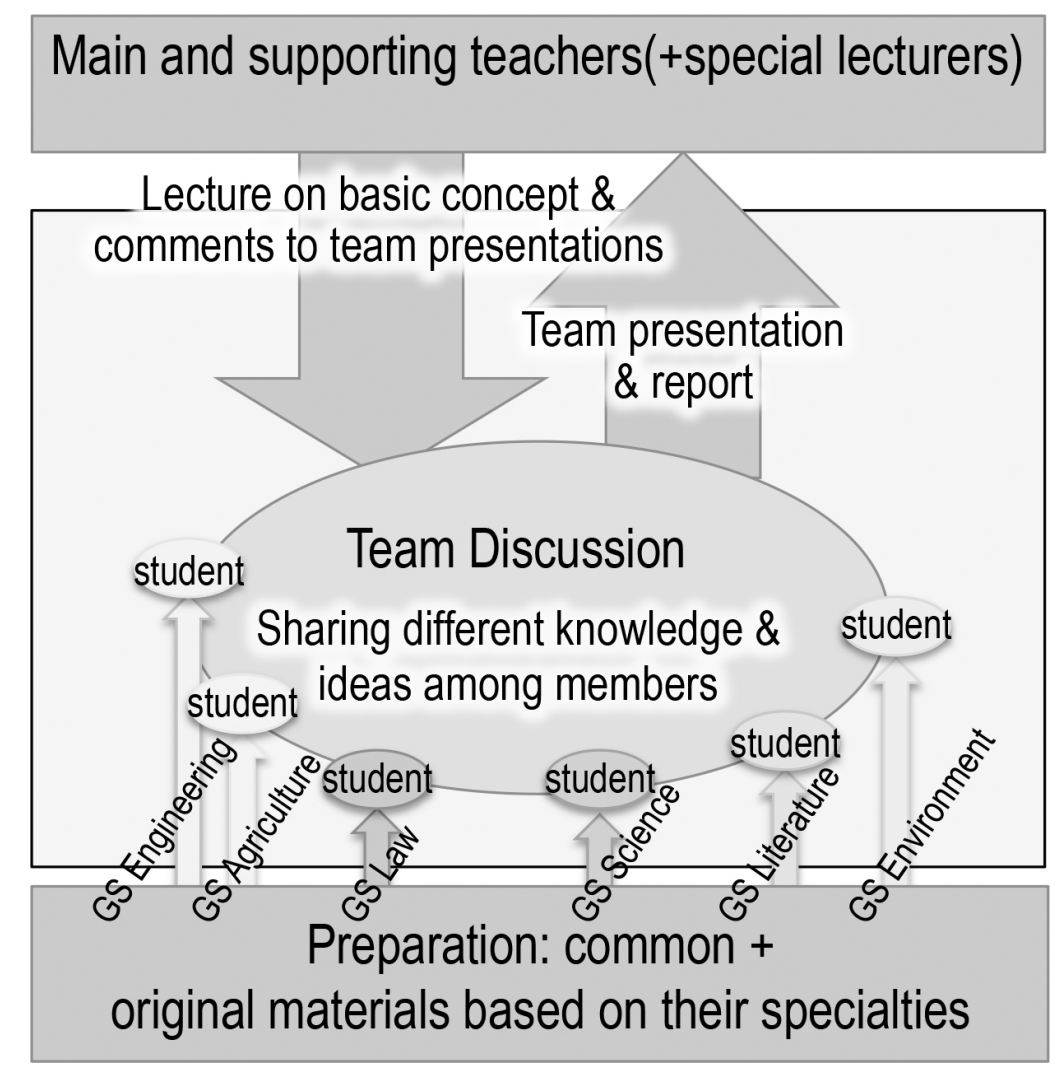

Fig.1 Team-based learning by students coming from various graduate school such as graduate school of Science (GS. Science).

\subsection{NITOBE Portfolio, Placement Test and Systems of Mentors and Advisors}

We have also introduced NITOBE Portfolio (NPF), placement test and systems of mentors and advisors, as mechanisms for promoting students' proactive learnings and quality assurance of Nitobe School. NPF enables the students to visualize their learning processes and progress. Using NPF system, students can record not only their coursework (exercises, reports, individual evaluations, etc.) and results of placement test in Nitobe School, but also course and research 
work in their graduate schools, their achievements, with their self-evaluation, advisors' comments etc. We have improved NPF from the academic year 2014 to the academic year 2016 on all functions are implemented on the web as e-portfolio (the detailed specification and usage of NPF reported in [16]). NPF also act as not only a tool to share information among students, supervisors and advisors etc., but also a recorded evidence. Therefore the students can concretely make self-evaluations and explain oneself based on not feeling but on recorded evidence, and timely to be advised and comprehensively to be judged by one's advisor etc. (Fig. 2). NPF is also able to work as IR as one of the side effects. Based on NPF as an IR tool, we conduct Plan-Do-Check-Act (PDCA) cycle of Nitobe School as quality assurance of education and collect records of their research activities for inter-comparison how to supervise students' research works. In undergraduate school, portfolio is usually used to check the status of student's activities of learning such as home works and attendances to lectures, and to prevent from students from leaving schools, as a tool for enroll management. However, NPF was designed to record two-way interaction between students and their supervisors, because research work in graduate school progress under their communication like apprenticeship system. Our largest purpose introducing NPF is to visualize research work as well as course work. NPF has the similar role in the field and experiment notes. If academic harassment by students' supervisors or misconducts unfortunately happened, record on NFP would work as evidence. After all graduate school implement NPF, as "big data," we will visualize the progress of student's research work before obtaining clear outputs such as presenting in academic meetings and submitting their papers, or give a warning or an alert before harassment happens as near miss. Therefore, NPF works effectively as an IR tool.

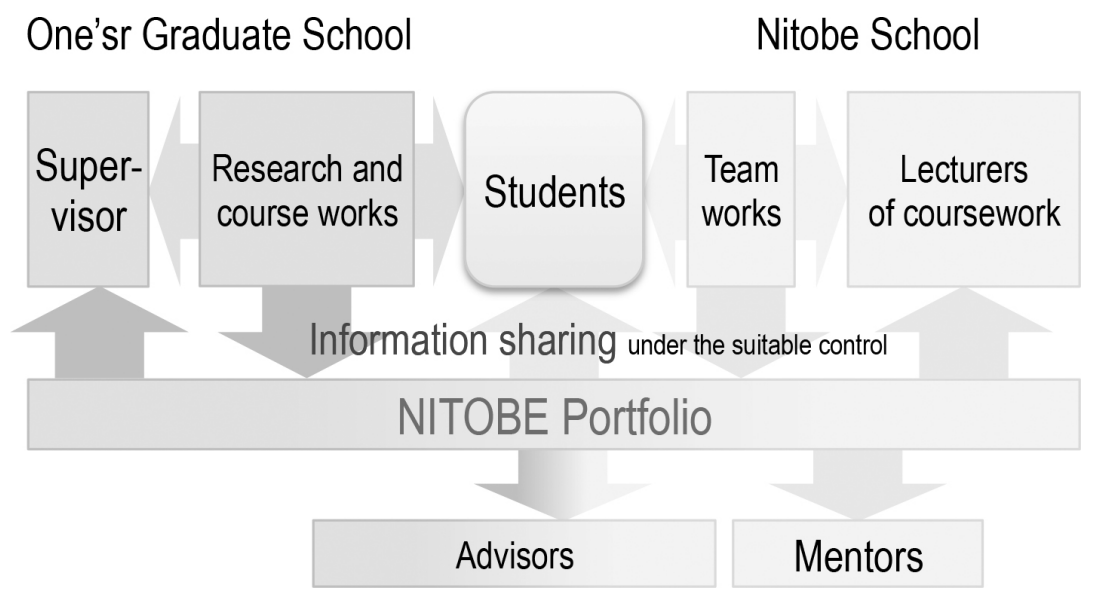

Fig.2 NITOBE Portfolio as a tool for information sharing

Placement tests are also conducted at students' enrollment and upon completion in Nitobe School, which are composed of diagnosis for Emotional Intelligence Quotient (e.g., Fundamental Competencies for Working Persons by Ministry of Economy, Trade and Industry) and efficiency in foreign language skills (e.g., TOEFL, TOEIC) as supplement of NPF in a viewpoint of objectivity. In the academic year 2014, we conducted that some graduate students 
as monitors take the three actual diagnosis and two foreign language skills: SEQ (Student Emotional Intelligence Quotient), PROG (Progress Report On Generic skills), and Career approach, TOEFL-ITP, and TOEIC-IP. We adopted SEQ in a viewpoint of suitability for international students, and both TOEFL-ITP and TOEIC-IP, because TOEFL is a world standard of English ability proof in the world in academic field and TOEIC is also well-known measurement for English skill in the business sector in Japan [17,18].

As advisors, professors in students' graduate school (or discipline close to students') act to support students to connect their specialties and " $3+1$ competencies". In other words, advisors will advise students comprehensively to understand their strengths, both for their specialties and as well as " $3+1$ competencies" based on NPF. As role models close to students, mentors are former graduates and alumni of Hokkaido University (in future, graduates from Nitobe School) in their early thirties, who will support students to make them aware of their carrier paths, help them broaden their views and build human networking, through short meetings and interaction with current students. The advantage is that the Nitobe School graduates will obtain worldwide personal relationships through such timely meetings. In this way, Nitobe School will become a gateway of society, and students and graduates will produce "Socio-Academic Ecosystem" connecting Hokkaido University and international society over disciplines and generation (Fig. $3)$.

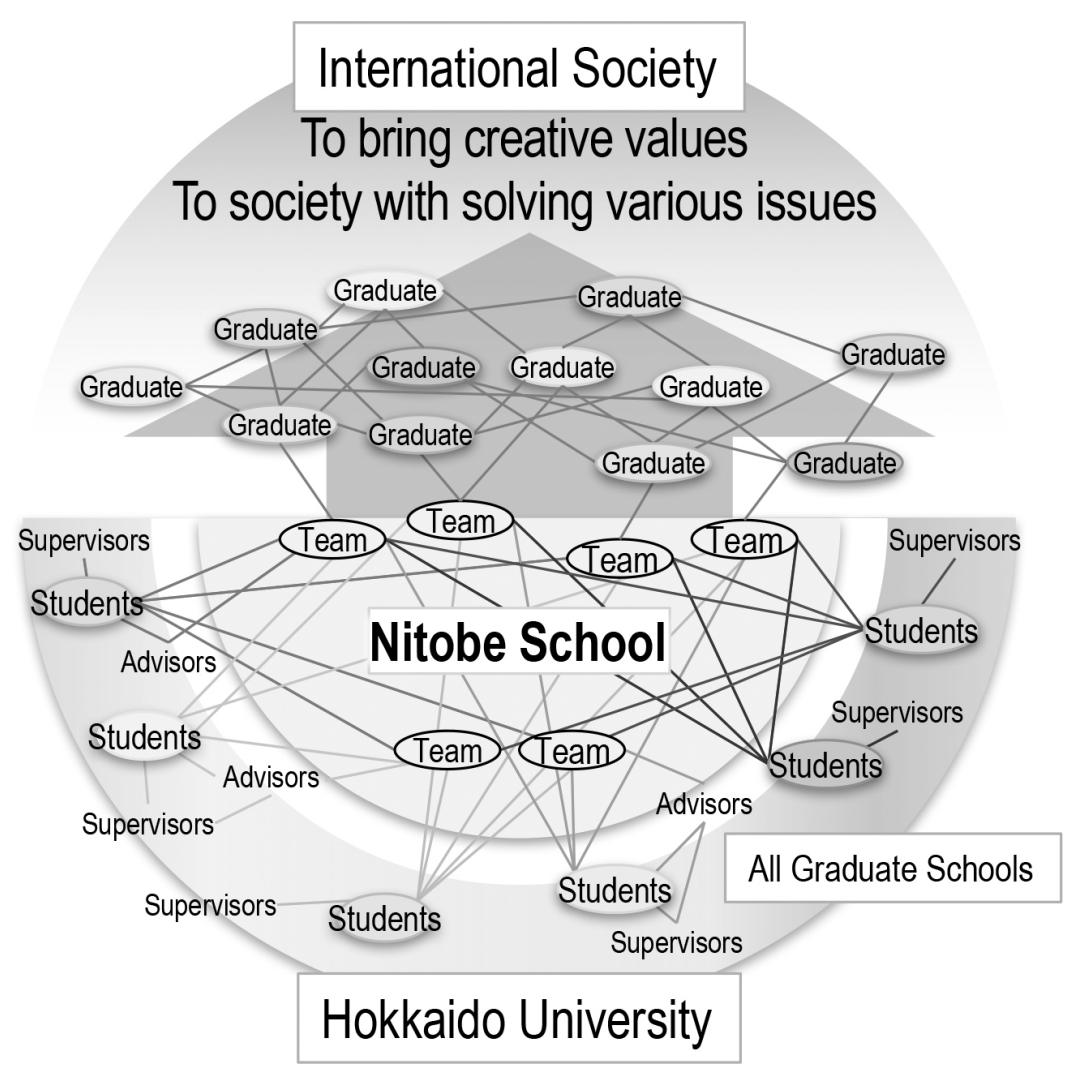

Fig.3 "Socio-Academic Ecosystem" connecting Hokkaido University and international society over disciplines.

Warden and Benshoff indicated as following: "Although students desire to do interdisciplinary work, the students and faculty in traditional departments are required to meet additional and 
often conflicting requirements" and "engagement in an interdisciplinary program may be seen by some as a career risk [19]" [11]. If students could not explain the relationship between their courses works in interdisciplinary program and their research works in traditional graduate school, they would face to a lack of understanding from their supervisors and other students in their graduate schools. In order to avoid and improve the difficult situations, both advisors and NPF will be a liaison connecting Nitobe School with students' graduate schools, and also present students' activities in Nitobe School to their supervisors. While "d.school" and "i.school" provide human network for graduates who share their minds cultivated by two schools, the human networking of Nitobe School is connected with advisor and their supervisors in their traditional graduate schools. The human networking is expected to disseminate the prototypes of Nitobe School such as " $3+1$ competencies" and NPF over all graduate schools.

\subsection{Main Coursework in Basic Program of Nitobe School}

Nitobe School offers the basic program mainly for the graduate students in master course (including professional graduate schools), however the graduate students in doctoral course can also enroll into Nitobe School. To confirm whether TBL under the concept of "microcosm of international society" works or not, we conducted a trial course consisting of eight times by three hours during two months in the academic year 2014, in which graduate students from many graduate schools took lecture classes with TBL. Based on this trial, we determined the structure of the basic program, which has the following main four courses with total eight credits, and each course is conducted in eight times by three hours in each quarter:

(1) Start-up Course: Learning basic skills for team-based activities such as facilitation, project management and professional ethics,

(2) Global Issues Course: introducing TBL as sharing information about global issues such as climate change and SDGs,

(3) Problem-Solving Course: Search and get the knowledge related to solving problems such as marketing, social research, and presenting their proposal

(4) Problem-Finding Course: Addressing and solving problems, also suggesting solutions out of classroom collaborated with theme-coordinated teachers, based on students' idea,

where students learn the team activity itself to solve the problem by team by step.

In the academic year 2015, each course has two classes of around 30 students, with several joint-sessions with mentors exchange meetings. Through these main four courses, five students per team were formed (team members are continuously shuffled for each course), and their team activities are built upon step-by-step to reach a higher level from the basic state for them to collaborate with people even outside the classroom. In each course, under the responsible professor who designs and conducts total coursework, two teachers (main and sub teachers) take charge of one class at times, special lecturer and theme-coordinated teachers will join in the class activities. Various teaching methods such as flipped classroom using e-learning and problem-solving learning (PSL) as project-based learning (PBL) were also implemented in each course. The detailed contents of main courses are as follows [20,21].

The Start-up Course serves as the foundation for all courses in the 2nd and subsequent quarters. It introduces students to the learning style of the Nitobe Program for Graduate Students and provides a solid foundation for TBL. Students will first be introduced to the basic study principles of the Nitobe Program for Graduate Students. They will then set their learning goals 
and seek to acquire advanced practical skills, which are required for study in the second and subsequent quarters. This course places emphasis on the development of the Competency in Continuous Personal Development and the Competency in Team Organization and Management, in particular. To develop the Competency in Team Organization and Management, students will intensively study through TBL in the first half of the course. They will acquire the basic team management skills through learning about facilitation, team building, team management and leadership theories. The latter half of the course will introduce students to professional ethics to determine whether achievements are socially acceptable or not. They will also learn the attitude of tackling difficult problems with team/project members through discussion with social entrepreneurs (invited from outside Hokkaido University to serve as instructors).

The Global Issues Course introduces students through TBL to a variety of global issues that must be tackled beyond the traditional academic boundaries. In the academic year 2015, the course will devote three classes to global warming issues and SDGs. In classes featuring global warming issues, for example, students will collect information in their fields of specialty students majoring in science will collect information on the mechanism of global warming, those in engineering on technical solutions, those in public policy and law on conventions and legal systems, and those in economics on carbon emission trading. The students will then make presentations on the collected information to other team members, and listening to the presentations delivered by other team members, understand the provided information before developing team opinions. This is intended to improve the Competency in Continuous Personal Development and the Competency in Team Organization and Management. The course also seeks to develop Professional Ethics and the Competency in Giving Back to Society by encouraging students to understand different standpoints such as those of developed countries vs. developing countries or producers vs. consumers.

In the Problem-Solving Course, students will seek to find solutions to the set problems using the PSL technique. That is, they will learn how to solve problems through teamwork. They will also learn how to run organizations and collaborate with others as necessary to solve problems to build team-oriented problem-solving competency (i.e., Competency in Team Organization and Management). This competency will naturally enhance their ability to tackle challenges to achieve set objectives as they continue to improve themselves by modifying plans as necessary (Competency in Continuous Personal Development). In their roles as highly educated specialists, students will have to identify social needs rather than trivializing problems as relating only to certain individuals or companies (Professional Ethics) and address these needs by creating new social values (Competency in Giving Back to Society). The course will devote attention to two problems, and students will work on each problem for four weeks to propose solutions. Students will make presentations on the solutions in a session attended by mentors who play active roles in society.

In the Problem-Finding Course, problems are not provided to students; they are expected to find them on their own and learn the processes up to arriving at solutions through teamwork. Using the PBL method, students will specifically learn the processes involved in finding a problem, analyzing its causes, investigating and assessing existing solutions, and proposing new solutions. To find problems, students will need curiosity, the desire for self-improvement and the willingness to take on difficult challenges (Competency in Continuous Personal Development). To analyze the problem to identify its causes, experts from different disciplines will have to join 
hands (Competency in Team Organization and Management). Students will also have to consider if the solutions they arrive at through teamwork will contribute to the wealth and wellbeing of society at large (Competency in Giving Back to Society and Professional Ethics). Therefore, by taking this course that will put finishing touches on the Basic Program, students will be able to improve their overall " $3+1$ Competencies", the aim of the Nitobe Program for Graduate Students. They will spend eight weeks to tackle a problem in a single team. Market research, interviews with theme-based instructors and other extracurricular activities away from the classroom are also planned so that students can propose a practical solution based on the analysis of the problem, not an armchair theory.

Global Issues Course dealt with subjects undergoing, and students learnt well with flipped classrooms and TBL, before adaptation of SDGs adapted by United Nations in September, 2015 and the Paris Agreement against global changes in December, 2015. Based on both skills of the team activities by Start-up Course and knowledge of global issues by Global Issues Course, students challenge the specific problems to make a proposal based on their survey as PSL or PBL in Problem-Solving Course and Problem-Finding Course. For students who learn English as a communication tool, English learning classes were supplementary provided in each quarter of 2015.

\subsection{Selection of participants and completion requirement}

Participants in the basic program are selected based on the following reason for applying to Nitobe School described in the application form as follows:

(1) applicants' backgrounds such as their studies in undergraduate schools and research work in graduate schools,

(2) their vision how to play a leading role in contributing to the development of international society after their graduation,

(3) how to develop your abilities according to competencies in " $3+1$ competencies."

Requirements for completion of the basic program are completions of (1) the main coursework above and (2) their master course at the graduate schools that they are affiliated for students in master course with (3) success in the program-completion-examination that involves self-assessment reports and other documents based on the Nitobe Portfolio.

\subsection{Advanced Program of Nitobe School}

In the advanced program, students' team will make projects to solve the issues under the collaboration with companies, local regions, etc. in domestic and overseas fields, which will be based on the basic program learning. The advanced program was to start in the academic year 2017 with around 25 students who completed the basic program for graduate students and are in their doctoral courses. At the start of the academic year 2017, the students without completion of the basic program were also accepted.

\section{Implementation of Nitobe School}


In the academic year 2015, Nitobe School started with 64 students selected from 118 applicants, who came from 17 graduate schools [21]. Total 46 graduate students obtained the certificate of Nitobe School program. The first priority of a program that seeks engagement was to recruit participants from various levels and areas of expertise [10]. As the point of diverse and engaged participants, we regarded Nitobe School as a quality graduate education, because Nitobe School successfully recruited participants coming from 17 graduate schools in the academic year 2015 . As the basic program is one year program however the completion of master course is also required. Total seven students, the second year in master course and doctor course, completed the program in the academic year 2015. With their diploma of master degree, 37 students of $1^{\text {st }}$ year of master course completed the program in the academic year 2016, and some students continue to study in master course due to studying abroad for one year. In the academic year 2016 (2017), 78 (107) students were, selected from 89 (116) applicants, participated in the basic program, and 25 students were, selected from 33 applicants, participated in the advanced program started in the academic year 2017.

Nitobe School gave the students a supportive push forward to respect and understand different discipline, nationalities, cultures, and preferred language. For example, in questionnaire to students taking in Problem-Finding Course, $80 \%$ of students answered that they learned many new things from team members gathering from different graduate schools, and $64 \%$ of students also answered that ideas of other team members influenced their perspectives [21]. Both of students and teachers regarded that taking courses were a good experience of TBL provided by Nitobe School [22]. Newswander and Borrego suggested that an interactive classroom setting is one in which "Students participate in learning activities in which they connect theoretical and applied knowledge to complex problems, issues, and situations in the real world [9]" and students are "more adept at translating and communicating theoretical and technical knowledge to others [9]" [10]. Based on interactive teaching and learning, a component of Haworth and Conrad's engagement theory of quality graduate education, Nitobe School provided an excellent classroom setting and discussion themes, in which students and teachers made interactive teaching and learning with TBL.

As a result, participating students were able to understand the importance of " $3+1$ competencies". Especially, "Microcosm of international society" worked well for students who were previously hesitant to communicate in English and discuss with students from diverse backgrounds in the team environment. The role of advisors also worked out well for keeping the quality assurance and helped a lot in collaborating and understanding with participating graduate schools. The 20 mentors in the academic year through their experience and interactions clearly provided information regarding society-focused abilities through discussions with fellow students and relating to their specialties and knowledge after received the degree from graduate schools.

We believe one year is too short to prepare the coursework as "microcosm of international society" and indeed needs many innovative mechanisms for promoting students' proactive learnings and bring about quality assurance of Nitobe School. Since some professors regarded the unfinished state as a failure, some trials will be not improved by PDCA-cycle but remains as an unfinished state. Especially, in order to realize "microcosm of international society", it is required for professors as well as students to deeply respect and understand key differences between several facts. With these skills and understandings a good platform can be built for faculty development and student guidance. In future, the workload of Professors in charge of 
Nitobe School should be decentralized while maintaining the quality of coursework and promoting system such as advisors are maintained. In technical aspect, it should be established for graduate students, in Graduate School of Fishery Science, participating from Hakodate Campus five hours off main campus to participate more easily.

According to PI at Site A reported in Newswander and Borrego, reflection and revision are especially important in new interdisciplinary fields, where methodological standards and knowledge bases are not yet established, NITOBE School need them to conduct PDCA-cycle based on the engagement theory. There is an allocation problem of resources: 5-years-fixed-time position for full time workers at Nitobe School and only a small number of professors working with their time management. The problem of adequate resources is the last component of engagement theory. At the long-termed viewpoint, our system such as advisors and NPF would present one solution for students who meet a conscious barrier between interdisciplinary and traditional educations suggested by Newswander and Borrego.

As mentioned in introduction, research and course works are inseparable in graduate schools. Students are expected to obtain abelities (specialty competencies) activating their specialties. However an academic field became the higher level and more subdivided, they can obtain less generic competencies (Fig. 4). Recently, although occasions to make research collaboration increase, the most of their collaborations are conducted within their discipline or close disciplines. Parcitipating these collaborations is an effective to deepen their specialties. Nitobe School was designed to provide the opportunities collaborating with people from various backgrounds to students, who obtain " $3+1$ competencies" that activate their specialty as a result. Participating Nitobe School is an effective opportunity for students to awake to the changing surround of their specialties; students can realize the development and significance of their research based on not only the inside of their discipline but also interdisciplinary field. As a result, students will make actions with the advantage of broader view.
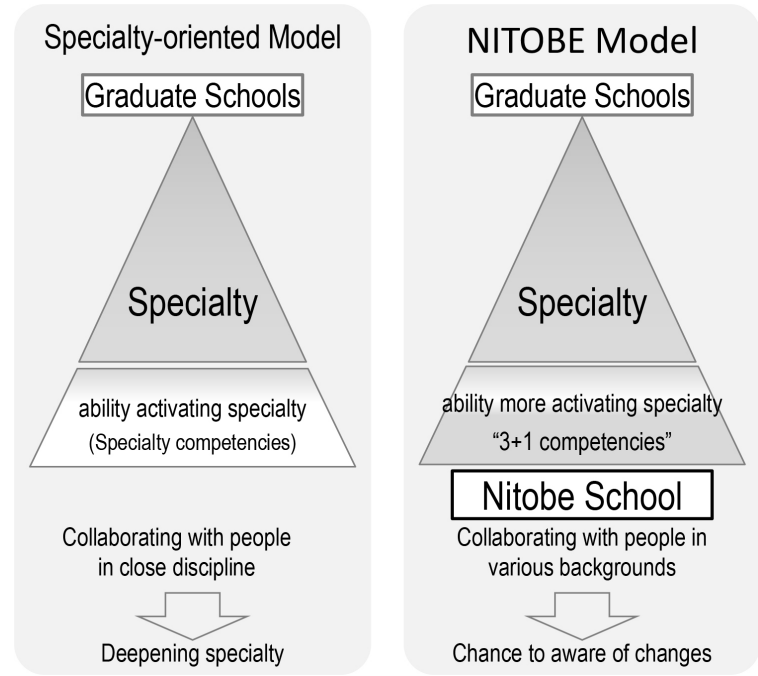

Fig. 4. Schematic illustration of traditional graduate school and Nitobe School

\section{Conclusion}


Using the advantage of Research University, Nitobe School was successful in providing the chance for graduate students to deeply understand the importance of " $3+1$ competencies" with regards to "microcosm of international society", in which graduate students from diverse nationalities and research backgrounds can learn with students from different discipline, nationalities, cultures, and in their preferred language with mechanisms to promote students' proactive learning and quality assurance. Our "microcosm of international society" is perfectly matched Haworth and Conrad's engaged theory of quality graduate education: diverse and engaged participants, and participatory cultures. From the long-termed viewpoint, our system such as advisors and NPF would present a solution for students to meet conscious barrier between interdisciplinary and traditional educations. In order to improve such learning environment, we should promote professors and faculty to deeply respect and understand differences beyond their expertise. That is, the target of human resources that correspond to the drastic change in international society and is possible through combined efforts from professors as well as graduate students

\section{Acknowledgement}

This paper was mainly based on "Fundamental Concept of Nitobe School" made by "Special Committee of Preparing Nitobe School (SCPNS)" in the academic year 2014, with supplemental information from conducting Nitobe School in the academic year 2015. Prof. Naoyuki Funamizu proposed the concept of "Socio-Academic Ecosystem", which was developed by a primal group of few interested professors (including authors) in the academic year 2013. Prof. Ryusuke Hatano and MS, one of authors, refined competency elements of " $3+1$ competencies", with other SCPNS members. As for Professional Ethics of " $3+1$ competencies", Prof. Nobuo Kurata contributed to other members' understanding ideas of ethics. Prof. Kurata also designed selection of participating in and requirement completing the basic program with YY. Nitobe Portfolio (NPF) was developed based on the portfolio used in Graduate School of Life Science with coordination of Prof. Hisashi Haga, and implemented by Prof. Yukinori Kobayashi with MS. Professors Toru Hagiwara and Yukinori Kobayashi with MS designed detailed system of advisors and mentors, and hosted meetings of interaction with the current students. Prof. Taro Yamauchi coordinated English learning classes assocated with main coursework. YY and MS wrote up "Fundamental Concept of Nitobe School", which including efforts above. Prof. Haga took a charge of the second deputy of vice-principal of NITOBE School to succeed YY.

Mr. Tetsuya Matsumoto in Center for Open Education supported to make e-learning contents with YY as used in Flipped classrooms in Global Issues Course. Professors Takaaki Inoue, Katsusuke Shigeta and Naohiro Iida had lectures to SCPNS member about Sapporo Agriculture College, Massive Open Online Course (MOOC) and a PBL-style course conducted in public a policy school in Hokkaido University, which was helpful to design Nitobe School. Dr. Shotaro Imai and Ankit A. Ravankar reported NPF and students' activities in Problem-Finding Course, as sub-teachers of Problem-Solving Course and Problem-Finding Course in 2015. Reviewers gave us information about previous studies and cases dealing with interdisciplinary education. English proofreading conducted by Ms. Eriko Ishida and Mr. Yong Seuk Park.

\section{References}


[1] National Institution for Academic Degrees and University Evaluation, "OverviewQuality Assurance System in Higher Education: JAPAN,” 2009, p. 28.

[2] Wikipedia, “Kanban”; https://en.wikipedia.org/wiki/Kanban

[3] Ministry of Economy, Trade and Industry, "Fundamental Competencies for Working Persons"; http://www.meti.go.jp/policy/kisoryoku/Fundamental Competencies for Working Persons.ppt

[4] Ministry of Education, Culture, Sports, Science and Technology, "Toward construction of the undergraduate education," 52pp, MEXT, 2008. (in Japanese)

[5] Japan Scoety for the Promotion of Science, “Top Global University Project," Apr. 2014; http://www.jsps.go.jp/j-sgu/gaiyou.html (in Japanese)

[6] Stanford University, “d.school”; https://dschool.stanford.edu/

[7] The university of Tokyo, "i.school”; http://ischool.or.jp/

[8] M. Ichiki, M. Suzuki, S. Mine, H. Saitoh, K. Sugawara, Y. Kanazawa and R. Naganawa, "Recent program of AIST Innovation School," $6{ }^{\text {th }}$ IIAI International Congress on Advanced Applied Informatics (IIAI-AAI), 2017.

[9] Haworth, J. G., and C. E.Conrad, "Emblems of quality in higher education: Developing and sustaining high-quality programs," Allyn and Bacon, Boston, 1997, p. 253.

[10] Newswander L. K. and M. Borrego, "Engagement in two interdisciplinary graduate programs," Higher Education, 58, 551-561, 2009.

[11] Warden, S., and Benshoff, J. M. "Testing the Engagement Theory of program quality in CACREP-Accredited counselor education programs," Counselor Education and Supervision, 51, 127-140, 2012.

[12] Hokkaido University, Future Strategy for the 150th Anniversary of Hokkaido University. 14pp, 2014.

[13] Y. Yamanaka and S. Mitsui, "A Comprehensive Course Introducing Environmental Science: Case Study of "Introduction to Environmental Science" as a Common Course in the Graduate School of Environmental Science," J. Higher Education and Lifelong Learning, vol. 24, pp. 117-130, 2017. (in Japanese)

[14] United Nations, "Sustainable Development Goals" http://www.un.org/ sustainabledevelopment/

[15] Bromme, "Beyond one's own perspective: The psychology of cognitive interdisciplinarity." In P. Weingart \& N. Stehr (Eds.), Practising interdisciplinarity (pp. 115-133). Toronto: University of Toronto Press, 2000.

[16] Shotaro Imai, Michiyo Shimamura, Hisashi Haga, Yukinori Kobayashi, Ankit Ravankar and Yasuhiro Yamanaka "Utilization of the Student e-Portfolio in the Graduate Education The case report of Nitobe School Program in Hokkaido University," $6{ }^{\text {th }}$ IIAI International Congress on Advanced Applied Informatics (IIAI-AAI), 2017. 
[17] M. Shimamura, S. Imai, A. A. Ravankar, Y. Yamanaka, "How Does the English Ability of the Student Change through the Postgraduate Education? - The Case Report of Nitobe School Program in Hokkaido University," $20165^{\text {th }}$ IIAI International Congress on Advanced Applied Informatics (IIAI-AAI), p. 574, 2016.

[18] M. Shimamura and Y. Yamanaka, "What is the most suitable English test for the Graduate School students in Research University of Japan? - The case report from the trial of "Nitobe School Program" in Hokkaido University," 20176 th $^{\text {th }}$ IIAI International Congress on Advanced Applied Informatics (IIAI-AAI), 2017.

[19] D. Rhoten and A. Parker, "Risks and Rewards of an Interdisciplinary Research Path," Science, 306, 2046, 2004.

[20] Nitobe School, “Student Handbook”, pp. 28, 2015.

[21] A. A. Ravankar, S. Imai, M. Shimamura, G. Chiba and T. E. Takasuka, "Problem-based Learning and Problem Finding Among University Graduate Students," J. Higher Education and Lifelong Learning, vol. 24, pp. 9-20, 2017.

[22] S. Imai, A. A. Ravankar, M. Shimamura, T. E. Takasuka, G. Chiba, Y. Yamanaka, "Effect of teaching abilities and attitude on learning outcomes in TBL class for graduate students", IIAI International Journal Series: International Institute of Applied Informatics (IIAI IJIRM), Vol. 1, No. 2, pp. 17-35, 2017. 